\title{
Comprehensive analysis of expression profiles of long non-coding RNAs with associated ceRNA network involved in gastric cancer progression
}

\author{
WEI GU ${ }^{1}$, JIA-HUI REN ${ }^{2}$, XIONG ZHENG ${ }^{1}$, XIAO-YING HU ${ }^{1}$ and MEI-JIE HU ${ }^{1}$ \\ ${ }^{1}$ Department of Gastroenterology, Ruijin Hospital, Luwan Branch, Shanghai Jiaotong \\ University School of Medicine, Shanghai 200025; ${ }^{2}$ Department of Gastroenterology, \\ Dapuqiao Community Health Service Center, Shanghai 200333, P.R. China
}

Received November 30, 2018; Accepted June 13, 2019

DOI: $10.3892 / \mathrm{mmr} .2019 .10478$

\begin{abstract}
Long non-coding RNAs (lncRNAs) play critical roles in the development and progression of cancers. The present study aimed to identify novel lncRNAs and associated microRNAs (miRNAs or miRs) and mRNAs in gastric cancer. Differentially expressed lncRNAs (DElncRNAs) and differentially expressed mRNAs (DEmRNAs) of 6 paired gastric cancer and normal tissues were identified using microarray. The DEmiRNAs between gastric cancer and the normal control tissues were identified using miRNA-seq data from Cancer Genome Atlas. Common DElncRNAs from the Cancer RNA-Seq Nexus database and circlncRNAnet database were analyzed. A DElncRNAs-DEmiRNAs-DEmRNAs network was constructed by target prediction. Functional enrichment analysis was employed to predict the function of DEmRNAs in the network. The correlation between the expression of DElncRNAS and DEmRNAs in the network was analyzed. The expression levels of several genes were validated by reverse transcription-quantitative polymerase chain reaction (RT-qPCR). A total of 1,297 DElncRNAs, 2,037 DEmRNAs and 171 DEmiRNAs were identified. Among the 4 lncRNAs common to the 3 datasets, prostate androgen-regulated transcript 1 (PART1) was selected for further analysis. The analysis identified 5 DEmiRNAs and 13 DEmRNAs in the PART1-mediated ceRNA network. The DEmRNAs in the ceRNA network were markedly enriched in cancer-related biological processes (response to hypoxia, positive regulation of angiogenesis and positive regulation of endothelial cell proliferation) and pathways (cGMP-PKG signaling pathway,
\end{abstract}

Correspondence to: Dr Mei-Jie Hu, Department of Gastroenterology, Ruijin Hospital, Luwan Branch, Shanghai Jiaotong University School of Medicine, 149 South Chongqing Road, Shanghai 200025, P.R. China E-mail: meijiehu0119@163.com

Key words: gastric cancer, tumorigenesis, long non-coding RNA, PART1, competing endogenous RNA
cAMP signaling pathway and proteoglycans in cancer). Out of the 13 DEmRNAs, 11 were positively associated with PART1. The downregulation of PART1, myosin light chain 9 (MYL9), potassium calcium-activated channel subfamily $\mathrm{M}$ alpha 1 (KCNMA1), cholinergic receptor muscarinic 1 (CHRM1), solute carrier family 25 member 4 (SLC25A4) and ATPase $\mathrm{Na}^{+} / \mathrm{K}^{+}$transporting subunit alpha 2 (ATP1A2) expression levels in gastric cancer was validated by RT-qPCR. On the whole, the current study identified a novel lncRNA and associated miRNAs and mRNAs that are involved in the pathogenesis of gastric cancer that may serve as potential therapeutic targets for the treatment of gastric cancer.

\section{Introduction}

Gastric cancer is one of the most commonly diagnosed and lethal types of cancer, corresponding to an estimated 951,600 new gastric cancer cases and 723,100 deaths worldwide in 2012 based on GLOBOCAN data (1). In Europe, gastric cancer is the fourth leading cause of cancer-related mortality and was associated with 107,000 (6.1\%) cancer-related deaths in 2012 (2). Therefore, there is an urgent need for the elucidation of the molecular mechanisms underlying gastric cancer and for the development of effective therapeutic strategies.

A large proportion of the genome in human cells is transcribed into RNA molecules that lack protein-coding capacity $(3,4)$. Of these, long non-coding RNAs (lncRNAs) are transcripts with lengths $>200 \mathrm{nt}$ (5) that play important roles in tumorigenesis and cancer progression (6-8). A growing number of gastric cancer-related lncRNAs have been identified in recent years. For example, the expression of the IncRNA BRAF-activated non-coding RNA (BANCR) was found to be upregulated in gastric cancer and to be associated with the patient survival rate (9). The expression of IncRNA LINC00857 has also been shown to be associated with a poor survival rate of patients with gastric cancer, and the downregulation of LINC00857 inhibits gastric cell proliferation (10). The downregulated expression of IncRNA LINC01939 has been shown to be associated with gastric cancer progression and a poor survival (11). According to the competing endogenous RNA (ceRNA) hypothesis (12), ceRNAs compete with mRNAs for binding to the same miRNA 
response elements (13). Recent studies have identified an increasing number of lncRNAs that function as ceRNAs, and play critical roles in the pathogenesis and progression of gastric cancer. For instance, lncRNAs, HOTAIR (14), SNHG6 (15), and NNT-AS1 (16), have been shown to be significantly upregulated in gastric cancer tissues and to function as oncogenes by competitively sponging miR-331-3p, miR-101-3p and miR-424 to regulate human epidermal growth factor receptor 2 (HER2), Zinc finger E-box-binding homeobox 1 (ZEB1) and E2F transcription factor 1 (E2F1), respectively. However, further studies are required to identify additional cancer-related lncRNAs, miRNAs and mRNAs.

In the present study, we analyzed the expression profiles of lncRNAs and mRNAs in gastric cancer tissues and adjacent normal tissues using microarray and analyzed miRNA expression profiles in gastric cancer and normal samples from The Cancer Genome Altas (TCGA) by miRNA-seq. We identified differentially expressed lncRNAs, mRNAs and miRNAs (designated as DElncRNAs, DEmRNAs and DEmiRNAs, respectively). Gastric cancer-related DElncRNAs that were common to the Cancer RNA-Seq Nexus (CRN) database and circlncRNAnet database were identified. A DElncRNAs-DEmiRNAs-DE mRNA ceRNA network was constructed by target prediction based on the ceRNA hypothesis (12). Gene Ontology (GO) and Kyoto Encyclopedia of Genes and Genomes (KEGG) pathway analyses were also performed to predict the functions of the identified DEmRNAs. In addition, the correlation between the expression of DElncRNAs and DEmRNAs in the ceRNA network was analyzed. The expression levels of DElncRNAs and DEmRNAs were validated by reverse transcription-quantitative polymerase chain reaction (RT-qPCR). Taken together, the present study identified novel gastric cancer-related lncRNAs, miRNAs and mRNAs and provided insight into the diagnosis and treatment of gastric cancer.

\section{Materials and methods}

Datasets and data preprocessing. We analyzed microarray data from our previous study [Vu et al (17)], which was generated from the global profiling of lncRNAs and protein-coding transcripts in 6 gastric cancer tissues and 6 pair-matched adjacent normal tissues using Arraystar Human LncRNA Microarray v2.0 (Arraystar, Rockville, MD, USA). The demographic and pathological characteristics of the patients are presented in Table I. DElncRNAs and coding transcripts between gastric cancer tissues and matched normal tissues were identified using the cut-off values fold change (FC) $\geq 2$ and adjusted $\mathrm{P}$-value $<0.05$.

In addition, DElncRNAs between gastric cancer tissues and normal tissues were obtained from CRN (http://syslab4. nchu.edu.tw/; adjusted P-value <0.01) and circlncRNAnet (18) (http://app.cgu.edu.tw/circlnc/; FC $\geq 2$, adjusted P-value <0.05), respectively. The DElncRNAs common among the microarray data, the CRN data and circlncRNAnet data were selected for further analysis.

miRNA sequence data of stomach cancer samples from TCGA database were downloaded from the Genomic Data Commons Data Portal (GDC, https://portal.gdc.cancer. gov/). DEmiRNAs between gastric cancer tissues and normal tissues were identified using the DESeq package (version 3.34.6, http://www.bioconductor.org/packages/release/
bioc/html/DESeq.html) with the cut-off values of $\mathrm{FC} \geq 2$ and false discovery rate $(\mathrm{FDR})<0.05$.

Construction of the DElncRNA-DEmiRNA-DEmRNA network. First, the integrated DEmiRNA-DEmRNA and DEmiRNA-DElncRNA pairs were predicted using MiRanda (http://www.microrna.org/microrna/home.do) and RNAhybrid (http://bibiserv.techfak.uni-bielefeld.de/rnahybrid/), respectively. The threshold values were set to energy <-20 and score $>150$ in MiRanda and energy $<-25$ in RNAhybrid. Only pairs that exhibited an inverse correlation and that were common to both the MiRanda and RNAhybrid results were selected as the final DEmiRNA-DEmRNA and DEmiRNA-DElncRNA pairs. Subsequently, the DElncRNA-DEmiRNA-DEmRNA network was constructed based on miRNA bridges using Cytoscape software (version 3.6.1).

Functional enrichment analysis. GO and KEGG pathway analyses were conducted to predict the functional roles of the DEmRNAs in the DElncRNA-DEmiRNA-DEmRNA network using DAVID 6.8 (https://david.ncifcrf.gov/).

Correlation analysis of DElncRNAs and DEmRNAs. To further analyze the DElncRNAs and DEmRNAs in the DElncRNA-DEmiRNA-DEmRNA network, expression correlation analysis between the DElncRNAs and DEmRNAs was performed by Pearson's Correlation Coefficient using Gene Expression Profiling Interactive Analysis (GEPIA, http://gepia. cancer-pku.cn/).

RT-PCR validation of DElncRNAs and DEmRNAs. A total of 15 cases of gastric cancer and adjacent tissues were used for RT-qPCR validation. The demographic and pathological characteristics of all the patients are presented in Table I. This study was approved by Medical Ethics Committee of Ruijin Hospital Luwan Branch Shanghai Jiaotong University School of Medicine. All patients provided written informed consent. Total RNA $(1 \mu \mathrm{g})$ was reverse transcribed into cDNA using First Strand cDNA Synthesis kit (Thermo Fisher Scientific, Inc.), the reactions and procedures were as follow: $1 \mu \mathrm{l}$ Random Primer, $1 \mu \mathrm{g}$ Total RNA and RNase-Free $\mathrm{ddH}_{2} \mathrm{O}$ was added to $12 \mu \mathrm{l}$ total volume and was incubated at $65^{\circ} \mathrm{C}$ for $5 \mathrm{~min}$, then $4 \mu \mathrm{l}$ 5X Buffer, $2 \mu \mathrm{l}$ dNTP Mix, $1 \mu \mathrm{l}$ Protector RNase Inhibitor, and $1 \mu l$ Transcriptase added. The expression levels of the selected DElncRNAs and DEmRNAs were validated by RT-PCR using the SYBR-Green PCR master mix kit (Applied Biosystems, Inc.). RT-qPCR was performed on an ABI QuantStudio ${ }^{\mathrm{TM}}$ 6 Flex System (Applied Biosystems, Inc.). Primer sequences for the amplification of target genes and the reference gene GAPDH are presented in Table II. The PCR protocol was: $95^{\circ} \mathrm{C}$ for $10 \mathrm{~min}, 45 \mathrm{cycles}$ of $95^{\circ} \mathrm{C}$ for $15 \mathrm{sec}$ and $60^{\circ} \mathrm{C}$ for $60 \mathrm{sec}$. To establish the melting curve, after the amplification reaction was completed, the reaction was performed at $95^{\circ} \mathrm{C}$ for $10 \mathrm{sec}$, $60^{\circ} \mathrm{C}$ for $60 \mathrm{sec}$, and $95^{\circ} \mathrm{C}$ for $15 \mathrm{sec}$, and slowly heating from $60^{\circ} \mathrm{C}$ to $99^{\circ} \mathrm{C}\left(+0.05^{\circ} \mathrm{C} / \mathrm{s}\right)$. The relative expression levels of the genes were measured based on 3 independent experiments and calculated using the $2^{-\Delta \Delta C q}$ method (19).

Statistical analysis. Statistical analyses were performed using SPSS 21.0 software (IBM Corp.). All experimental data are 
Table I. Demographic and clinicopathological information of the patients.

\begin{tabular}{|c|c|c|c|c|}
\hline Feature & Group $(n=21)$ & $\begin{array}{c}\text { Microarray } \\
\text { assay }(n=6)(\%)\end{array}$ & $\begin{array}{l}\text { Reverse transcription-quantitative } \\
\text { PCR }(\mathrm{n}=15)(\%)\end{array}$ & $\mathrm{P}$-value \\
\hline \multirow[t]{3}{*}{ Age } & & & & 0.94 \\
\hline & $<60$ & $3(14.3)$ & $9(42.9)$ & \\
\hline & $\geq 60$ & $3(14.3)$ & $6(28.5)$ & \\
\hline \multirow[t]{3}{*}{ Sex } & & & & 0.61 \\
\hline & Female & $2(9.5)$ & $5(23.8)$ & \\
\hline & Male & $4(19)$ & $10(47.6)$ & \\
\hline \multirow[t]{3}{*}{ Differentiation } & & & & 0.83 \\
\hline & Low & $2(9.5)$ & $6(28.5)$ & \\
\hline & Middle and high & 4 (19) & $9(42.9)$ & \\
\hline \multirow[t]{3}{*}{ TNM } & & & & 0.82 \\
\hline & $\mathrm{T} 1+\mathrm{T} 2$ & $1(4.8)$ & $5(23.8)$ & \\
\hline & $\mathrm{T} 3+\mathrm{T} 4$ & $5(23.8)$ & $10(47.6)$ & \\
\hline \multirow[t]{3}{*}{ Stage } & & & & 0.73 \\
\hline & I+II & $3(14.3)$ & $8(38.1)$ & \\
\hline & III & $3(14.3)$ & $7(33.3)$ & \\
\hline \multirow[t]{3}{*}{ Metastasis } & & & & 0.83 \\
\hline & No & $4(19)$ & $9(42.9)$ & \\
\hline & Yes & $2(9.5)$ & $6(28.5)$ & \\
\hline
\end{tabular}

Table II. Primer sequences used for reverse transcription-quantitative PCR.

\begin{tabular}{|c|c|c|c|}
\hline Gene name & Primer sequence & Annealing temperature $\left({ }^{\circ} \mathrm{C}\right)$ & Aim band length (bp) \\
\hline $\mathrm{B} 2 \mathrm{M}$ & $\begin{array}{l}\text { F: 5'-CTCTTTCTGGCCTGGAGGCTAT-3' } \\
\text { R: 5'-AGTCAACTTCAATGTCGGATGGAT-3' }\end{array}$ & 60 & 135 \\
\hline PART1 & $\begin{array}{l}\text { F: 5'-CAAACGCCTGAGTGCTGCTG-3' } \\
\text { R: 5'-AGTAAAGGGGGTAACAATTCTAACA-3' }\end{array}$ & 60 & 90 \\
\hline MYL9 & $\begin{array}{l}\text { F: 5'-GCCTCGCTGGGTTTCATCC-3' } \\
\text { R: 5'-CGGTACATCTCGTCCACTTCCT-3' }\end{array}$ & 60 & 95 \\
\hline KCNMA1 & $\begin{array}{l}\text { F: 5'-GGACTTAGGGGATGGTGGTTG-3' } \\
\text { R: 5'-GGTTGGTGATGACATACCTCTTTG-3' }\end{array}$ & 60 & 146 \\
\hline CHRM1 & $\begin{array}{l}\text { F: 5'-TGGGACATGCCAATCACTGG-3' } \\
\text { R: 5'-CTTCCTCCAGGCACGCTACA-3' }\end{array}$ & 60 & 198 \\
\hline SLC25A4 & $\begin{array}{l}\text { F: 5'-CTATGATACTGCCAAGGGGATG-3' } \\
\text { R: 5'-TACATAATATCGGCCCCTTTCC-3' }\end{array}$ & 60 & 171 \\
\hline ATP1A2 & $\begin{array}{l}\text { F: 5'-TGGCAATGGATGACCACAAG-3' } \\
\text { R: 5'-CAGGTCCACTTGGTATTTGCG-3' }\end{array}$ & 60 & 62 \\
\hline
\end{tabular}

F, forward; R, reverse.

presented as the mean values \pm standard deviation. Differences between the gastric cancer group and normal group were determined using the Student's t-test. The comparisons between the clinicopathological characteristics of the patients used in the microarray and those used for RT-qPCR were carried out using the Chi-square test. Values of $\mathrm{P}<0.05$ and $\mathrm{P}<0.001$ were considered to indicate statistically significant and highly statistically significant differences, respectively.

\section{Results}

Identification of DElncRNAs, DEmRNAs and DEmiRNAs. A total of 1,297 DElncRNAs and 2,037 DEmRNAs were identified between the gastric cancer tissues and matched normal control tissues with the cut-off values $\mathrm{FC} \geq 2$ and P-value $<0.05$. A total of 171 DEmiRNAs were identified between the gastric cancer and normal control samples 


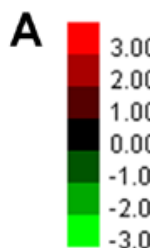

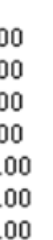
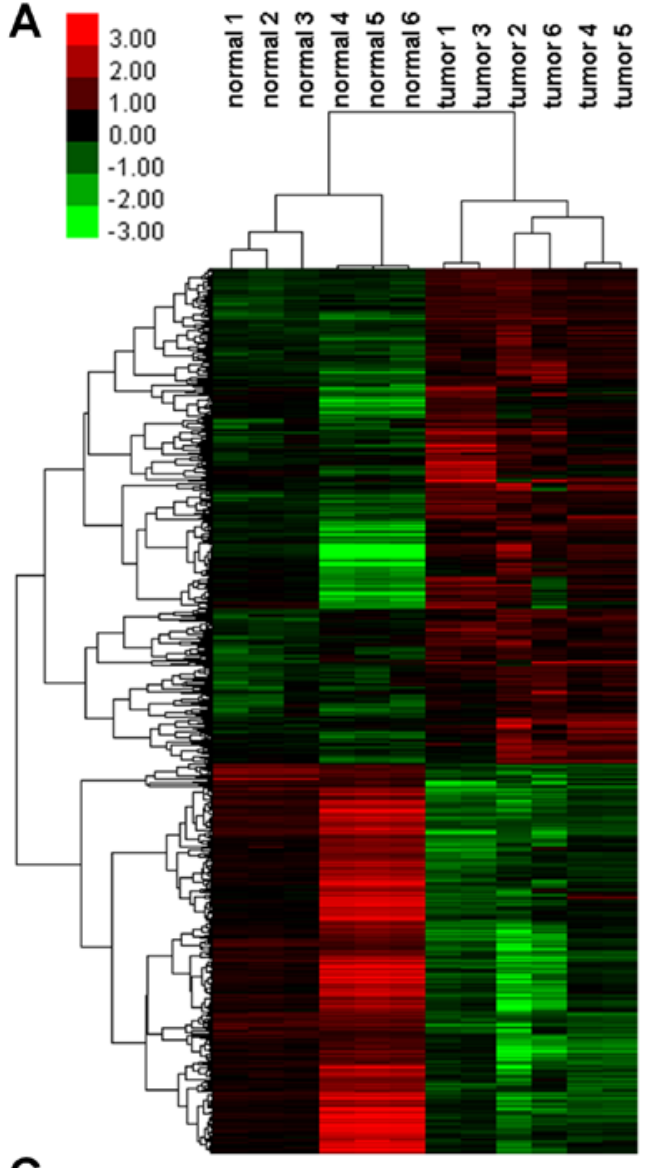

C
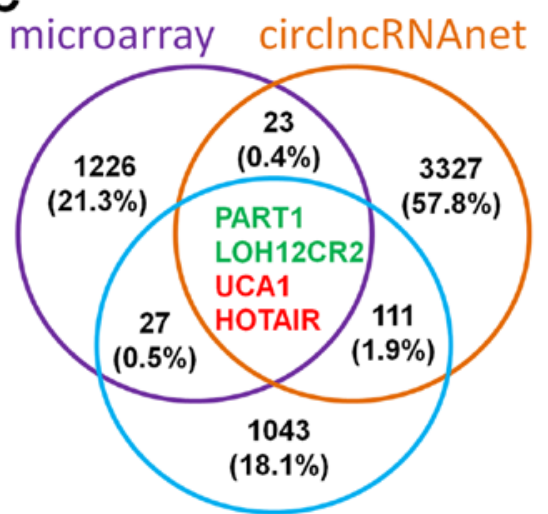

Cancer RNA-Seq Nexus

D
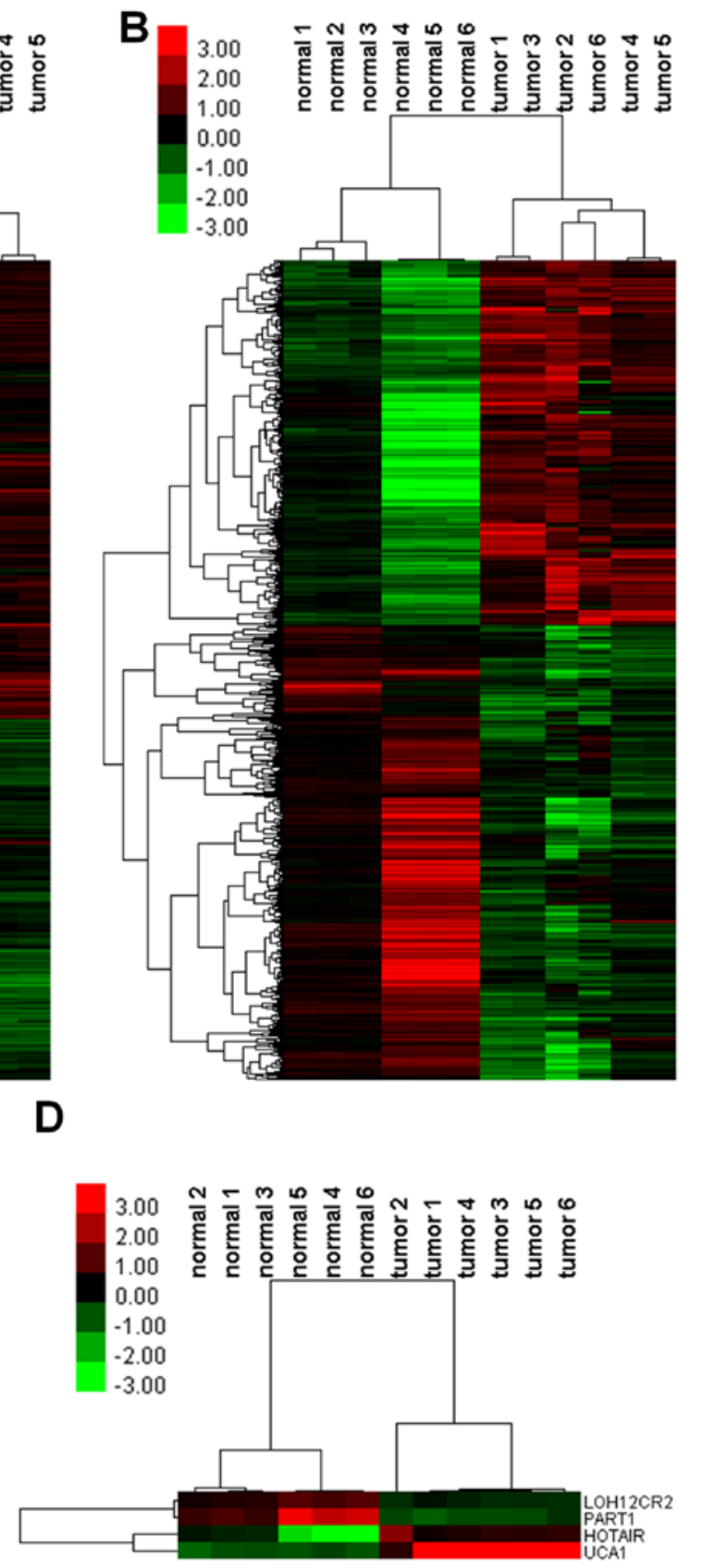

Figure 1. DElncRNAs and mRNAs between gastric cancer tissues and normal tissues. Heatmaps of (A) DElncRNAs and (B) DEmRNAs in gastric cancer. (C) The lncRNAs common among microarray, Cancer RNA-Seq Nexus (CRN), and circlncRNAnet data. (D) Heatmap showing the expression of the common lncRNAs. The red and green colors indicate upregulation and downregulation, respectively. DE, Differentially expressed.

from the TCGA dataset with the cut-off values $\mathrm{FC} \geq 2$ and FDR $<0.05$. The heatmaps for the total DElncRNAs and DEmRNAs are presented in Fig. 1A and B, respectively. A total of 4 lncRNAs [LOH12CR2, prostate androgen-regulated transcript 1 (PART1), HOTAIR and UCA1] were common among the microarray data, CRN data and circlncRNAnet data (Fig. 1C). LOH12CR2 and PART1 were markedly downregulated, whereas HOTAIR and UCA1 were significantly upregulated in the gastric cancer tissues. The heatmap showing the 4 overlapping lncRNAs is presented in Fig. 1D. Raw microarray data were deposited in the Gene Expression Omnibus database under the accession number GSE53137.
Pan-cancer expression analysis of IncRNA PART1. Literature mining identified HOTAIR and UCA1 to be associated with gastric cancer. PART1 has been demonstrated to play important roles in other types of cancer. In the TCGA datasets, the PART1 expression levels were markedly downregulated in various cancers, including bladder urothelial carcinoma (BLCA), breast invasion carcinoma (BRCA), head and neck squamous cell carcinoma (HNSC) and stomach adenocarcinoma (STAD) (Fig. 2), thereby suggesting its important role in tumorigenesis. However, the expression of LOH12CR2 exhibited no marked differences between the cancer and adjacent tissue (Fig. S1). Thus, PART1 was selected for subsequent analysis. 


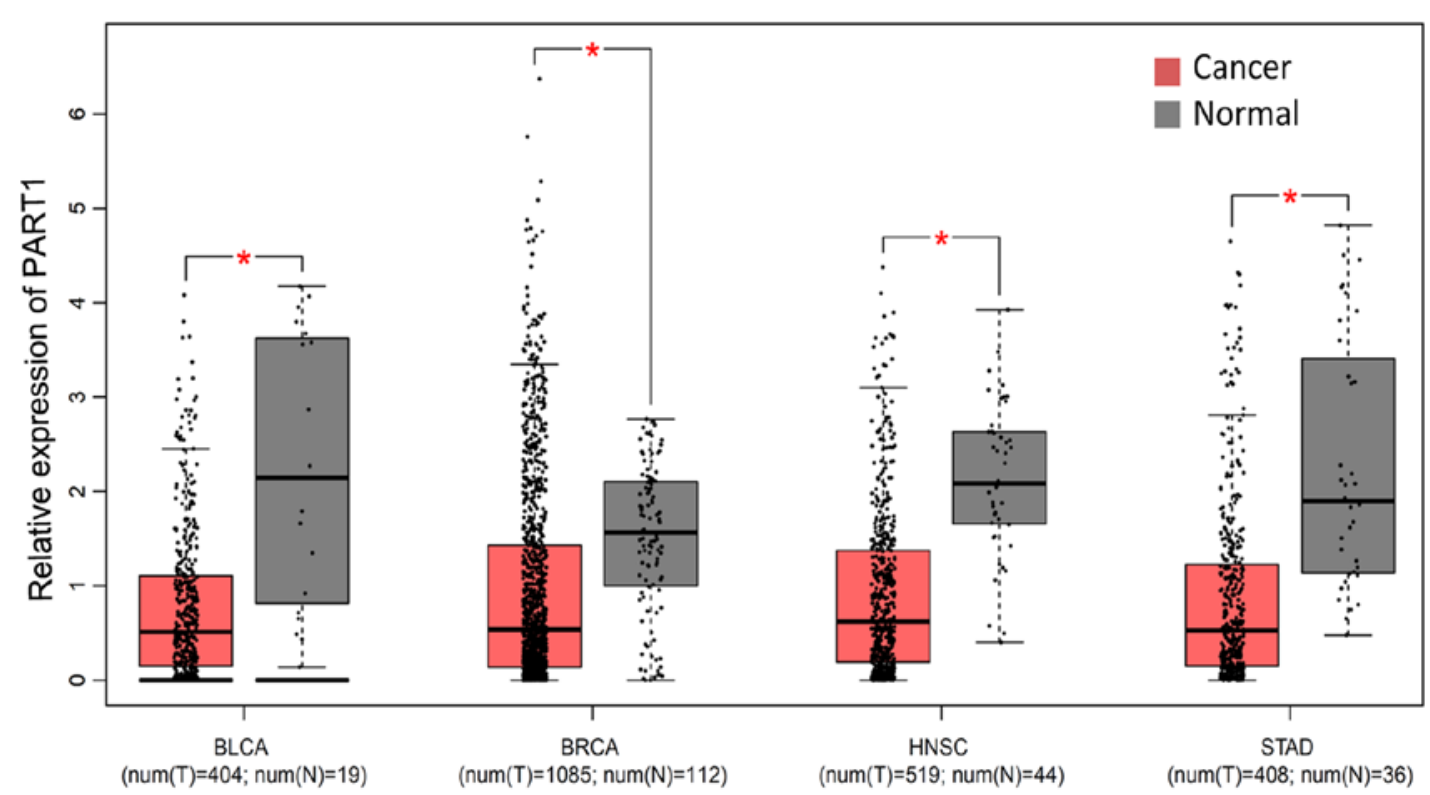

Figure 2. Expression of lncRNA PART1 in various types of cancer. PART1, prostate androgen-regulated transcript 1; BLCA, bladder urothelial carcinoma; BRCA, breast invasion carcinoma; HNSC, head and neck squamous cell carcinoma; STAD, stomach adenocarcinoma. "P<0.05.

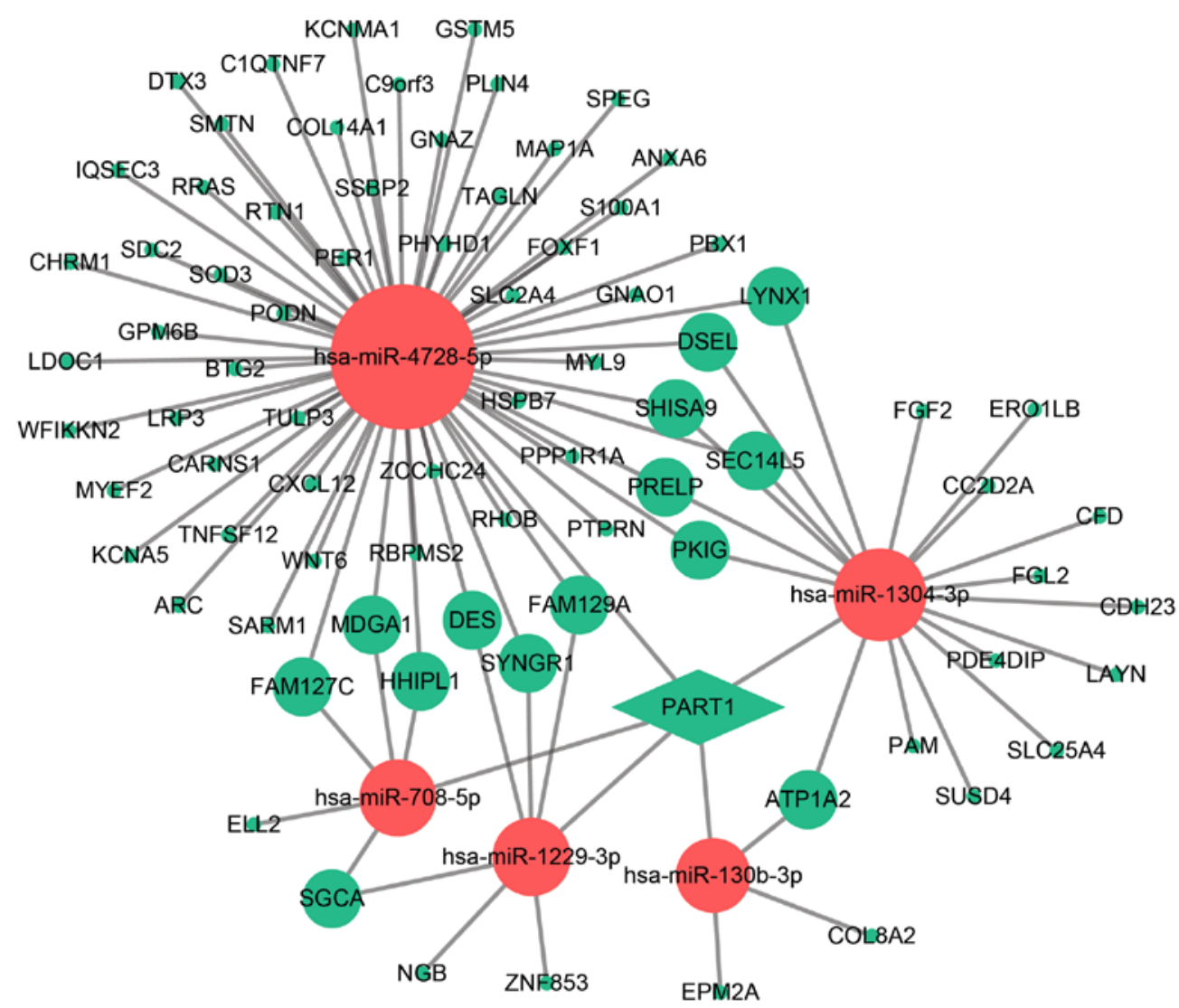

Figure 3. The PART1-DEmiRNAs-DEmRNAs ceRNA network. Green diamonds, green balls and red balls represent downregulated lncRNAs, downregulated DEmRNAs, and upregulated DEmiRNAs, respectively. DE, differentially expressed.

Construction of PART1-DEmiRNA-DEmRNA network. The PART1-mediated ceRNA network was constructed based on target prediction. As shown in Fig. 3,PART1 was found to interact with 5 upregulated DEmiRNAs, including hsa-miR-4728-5p, hsa-miR-1304-3p, hsa-miR-708-5p, hsa-miR-1229-3p and
hsa-miR-130b-3p. A total of 79 downregulated DEmRNA nodes were included in the network.

Functional enrichment analysis of the PART1-DEmiRNA$D E m R N A$ network. GO and pathway enrichment analyses were 
A

GO analysis

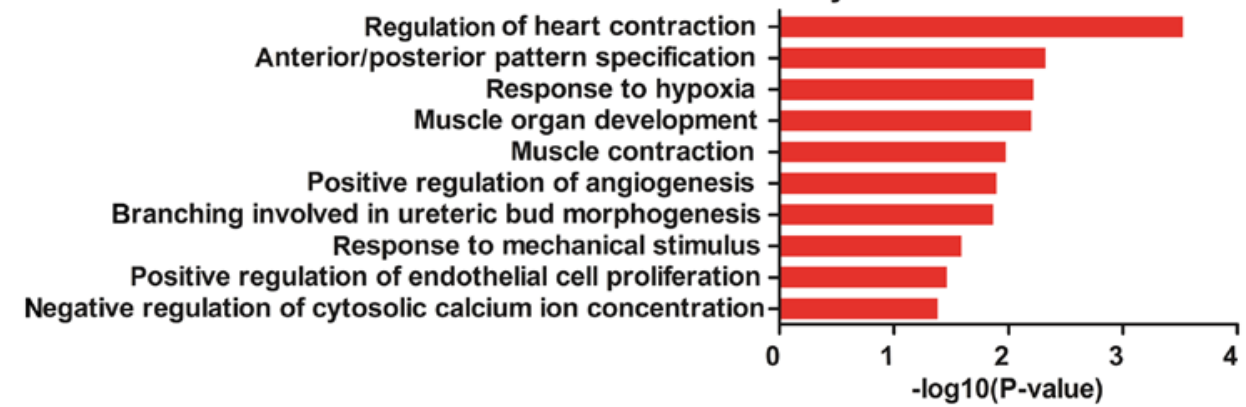

B

Pathway analysis

cGMP-PKG signaling pathway cAMP signaling pathway Proteoglycans in cancer Regulation of actin cytoskeleton Rap1 signaling pathway Long-term depression Arrhythmogenic right ventricular cardiomyopathy (ARVC)
HTLV-I infection Hypertrophic cardiomyopathy (HCM) Dilated cardiomyopathy

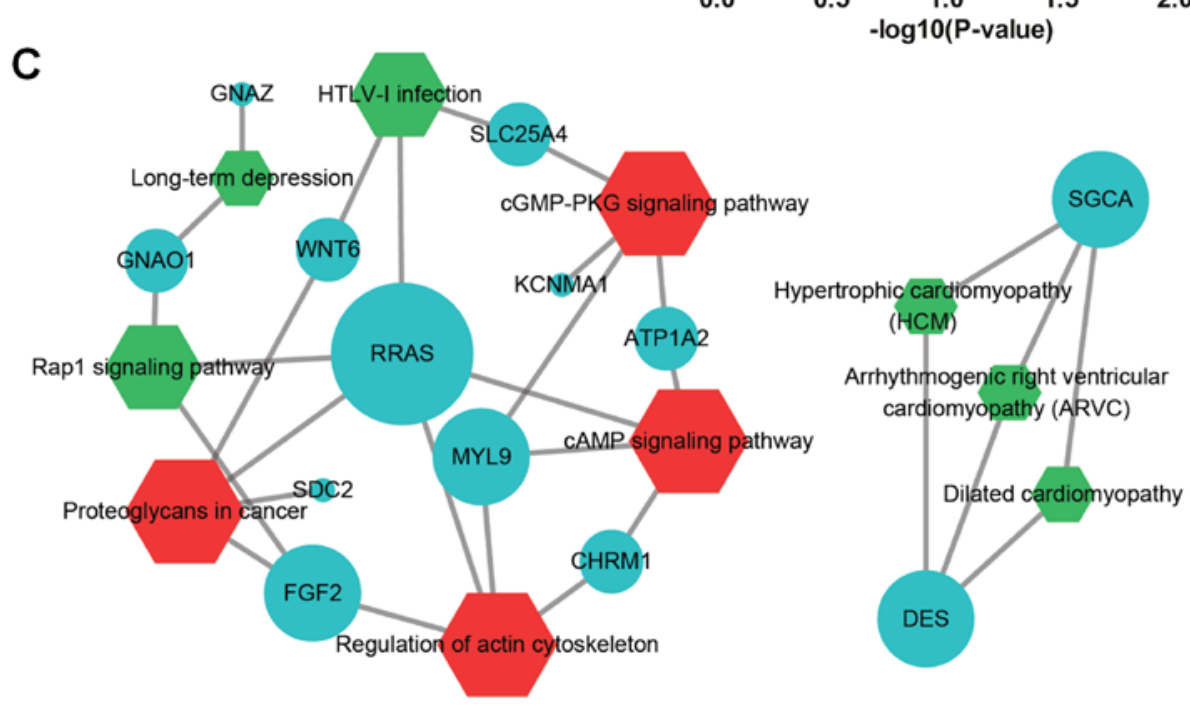

Figure 4. Gene Ontology (GO) and Kyoto Encyclopedia of Genes and Genomes (KEGG) pathway analysis. The top 10 (A) GO and (B) KEGG pathway terms enriched in the DEmRNAs in the ceRNA network. (C) DEmRNAs enriched in the top 10 pathway terms. Red color indicates statistically significant values (P-value $<0.05$ ), while the green color indicates non-significant terms (P-value $>0.05$ ). Hexagons represent KEGG pathways. Blue balls indicate enriched downregulated DEmRNAs. DE, differentially expressed.

performed to predict the functional role of the DE genes in the PART1-mediated ceRNA network and analyze the DEmRNAs in the ceRNA network. The top $10 \mathrm{GO}$ terms and top 10 pathway terms are presented in Fig. 4A and B, respectively. In particular, 4 pathways, namely the cGMP-PKG signaling pathway, cAMP signaling pathway, proteoglycans in cancer and regulation of actin cytoskeleton, were significantly enriched. The 13 identified DEmRNAs were enriched in the top 10 pathway terms (Fig. 4C).

Correlation analysis of PART1 and DEmRNAs. Expression correlation analysis was performed between PART1 and 13 DEmRNAs in the top 10 pathway terms. The results revealed that the expression levels of 11 out of 13 DEmRNAs were positively associated with PART1 expression levels in gastric cancer (Fig. 5). However, the expression of Wnt family member 6 (WNT6) and cholinergic receptor muscarinic 1 (CHRM1) exhibited no correlation with PART1 in gastric cancer (Fig. S2).
RT-qPCR validation of PART1 and associated DEmRNAs. To validate the reliability of the microarray results and the above-mentioned analysis, the expression levels of PART1, myosin light chain 9 (MYL9), potassium calcium-activated channel subfamily M alpha 1 (KCNMA1), CHRM1, solute carrier family 25 member 4 (SLC25A4) and ATPase $\mathrm{Na}^{+} / \mathrm{K}^{+}$ transporting subunit alpha 2 (ATP1A2) were confirmed by RT-qPCR. Microarray results revealed that the PART1 lncRNA and 5 candidate DEmRNAs were downregulated in the gastric cancer tissues compared with the adjacent normal tissues (Fig. 6A). Consistent with these findings, the results of RT-qPCR revealed that the relative expression levels of PART1, MYL9, KCNMA1, CHRM1, SLC25A4 and ATP1A2 were significantly lower in the gastric cancer tissues compared with the adjacent normal tissues (Fig. 6B). Moreover, another 7 downregulated DEmRNAs including G protein subunit alpha Z (GNAZ), G protein subunit alpha O1 (GNAO1), related 

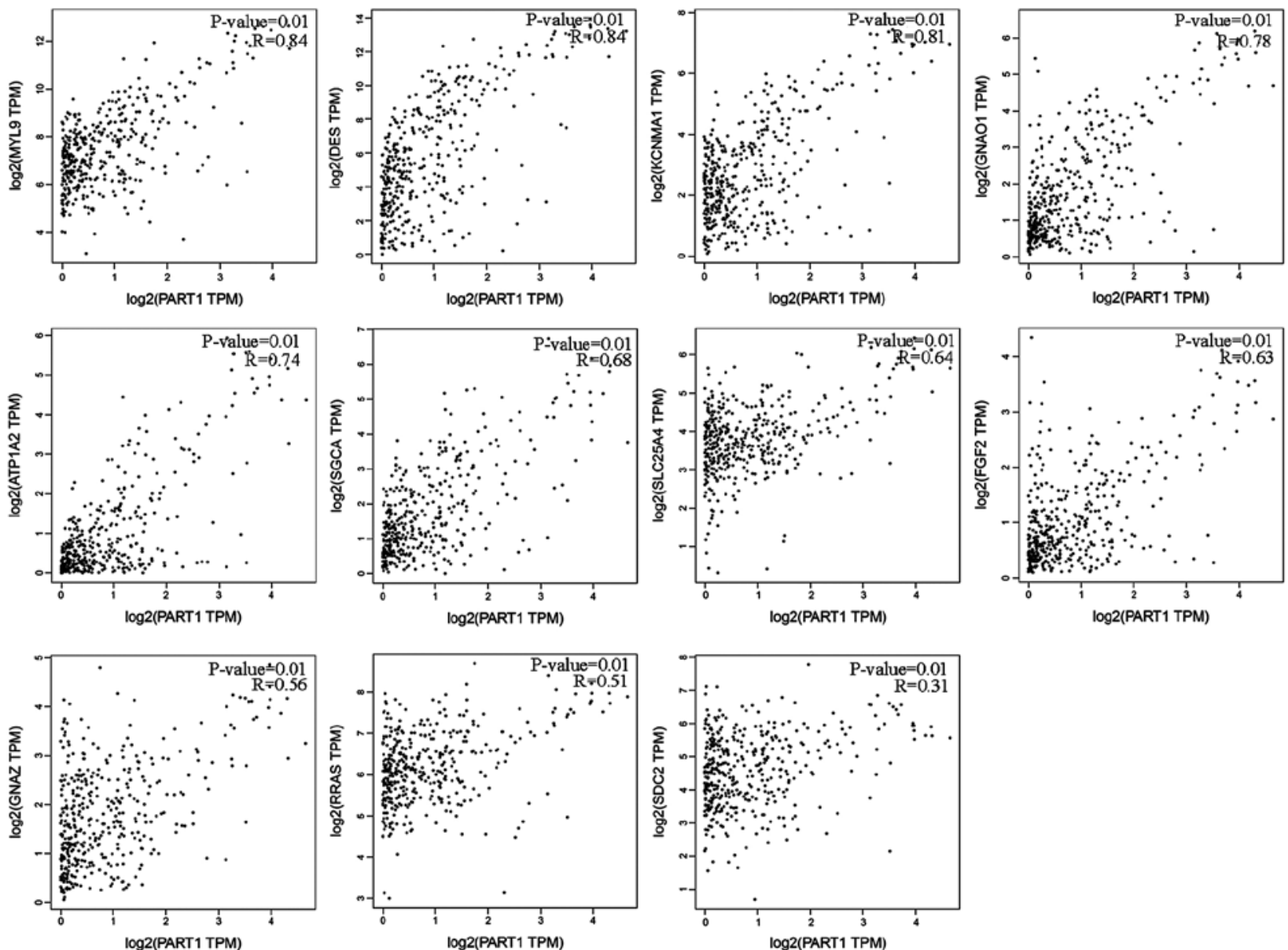

Figure 5. Expression correlation analysis for lncRNA PART1 and associated DEmRNAs. Horizontal axis indicates normalized PART1 expression levels; vertical axis indicates normalized DEmRNA expression levels. DE, Differentially expressed. MYL9, myosin light chain 9; DES, desmin; KCNMA1, potassium calcium-activated channel subfamily M alpha 1; GNAO1, G protein subunit alpha O1; ATP1A2, ATPase Na ${ }^{+} \mathrm{K}^{+}$transporting subunit alpha 2; SGCA, sarcoglycan alpha; SLC25A4, solute carrier family 25 member 4; FGF2, fibroblast growth factor 2; GNAZ, G protein subunit alpha Z; RRAS, related RAS viral (R-Ras) oncogene homolog; SDC2, syndecan 2.
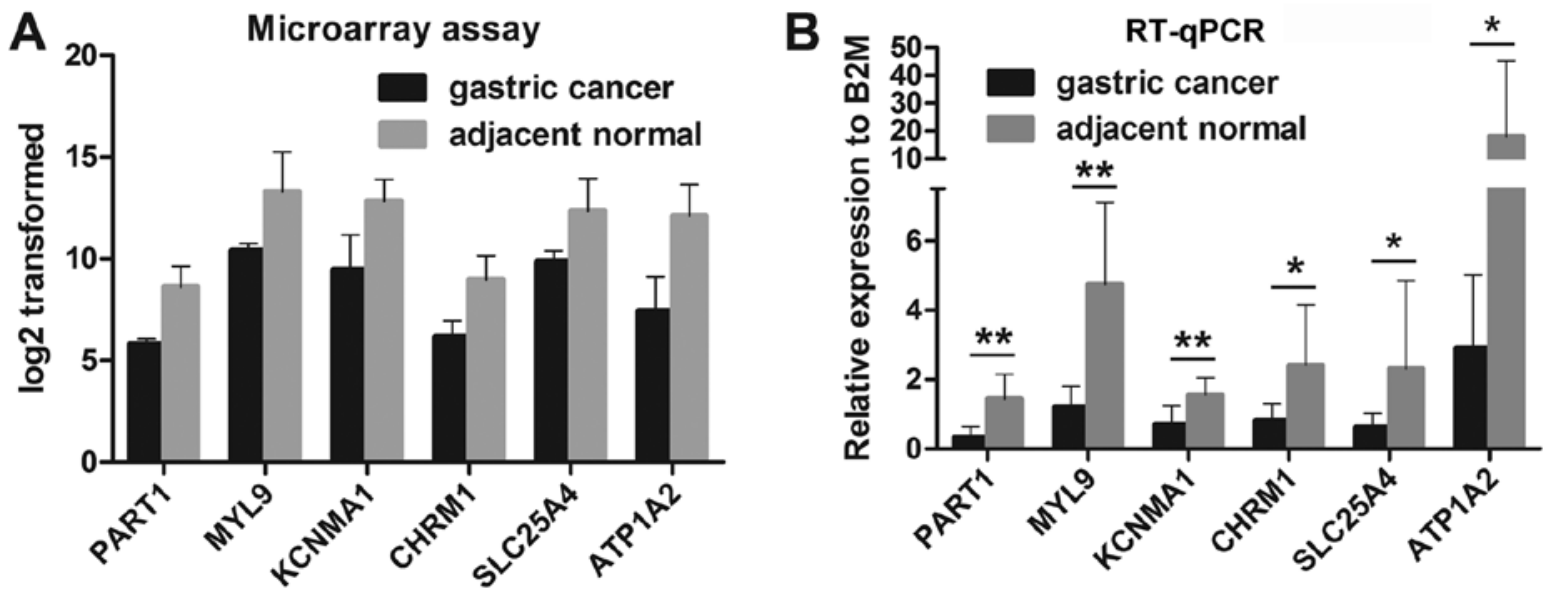

Figure 6. Expression levels of PART1 lncRNA and associated DEmRNAs in gastric cancer tissues and adjacent normal tissues based on (A) microarray results and (B) RT-qPCR. ${ }^{*} \mathrm{P}<0.05$ and ${ }^{* *} \mathrm{P}<0.001$, statistically significant differences and highly statistically significant differences, respectively. DE, differentially expressed.

RAS viral (R-Ras) oncogene homolog (RRAS), syndecan 2 (SDC2), fibroblast growth factor 2 (FGF2), sarcoglycan alpha (SGCA) and demsin (DES) in gastric cancer tissues relative to the adjacent normal tissues was confirmed by RT-PCR (Fig. S3).

\section{Discussion}

In the present study, we identified 1,297 DElncRNAs, 2,037 DEmRNAs, and 171 DEmiRNAs between gastric cancer tissues and matched normal tissues. Two upregulated lncRNAs 
(HOTAIR and UCA1) and two downregulated lncRNAs (LOH12CR2 and PART1) were common between the microarray, CRN and circlncRNAnet results. Several studies have previously reported that HOTAIR is an oncogene that plays a crucial role in the progression of various types of cancer (20), including gastric cancer $(21,22)$, lung cancer $(23)$, colorectal cancer (24), liver cancer (25), gallbladder cancer (26), breast cancer (27), cervical cancer (28), ovarian cancer (29), and prostate cancer (30). The oncogene UCA1 is known to be involved in various types of cancer, such as gastric cancer (31), breast cancer (32), bladder cancer (33) and colorectal cancer (34). In this study, GEPIA analysis of the TCGA data revealed that PART1 expression was significantly downregulated in various types of cancer, including gastric cancer. Thus, PART1 was selected for further analysis.

The PART1-mediated PART1-DEmiRNA-DEmRNA ceRNA network was constructed. PART1 was found to directly interact with 5DEmiRNAs(hsa-miR-4728-5p, hsa-miR-1304-3p, hsa-miR-708-5p, hsa-miR-1229-3p and hsa-miR-130b-3p) and to indirectly interact with 79 DEmRNAs via DEmiRNAs. miR-1229 expression was significantly upregulated in breast cancer and has been demonstrated to promote tumor growth by activating the Wnt/ $\beta$-catenin signaling pathway (35). miR-130b-3p upregulation is mediated by a mechanism that is dependent on the cAMP-response element binding protein and is known to promote thyroid adenomas development (36). miR-4728-5p expression has been shown to be significantly upregulated in patients with gastric cancer who are positive for HER2, which is overexpressed in gastric cancer and serves as a clinical target for gastric cancer therapy $(37,38)$. miR-708-5p is involved in various processes associated with tumorigenesis and acts as an oncogene in various cancers, including bladder cancer $(39,40)$ and colorectal cancer $(41)$. Taken together, the above-mentioned findings indicate that PART1 plays crucial roles in cancer.

Additionally, DEmRNAs in the generated PART1-mediated ceRNA network were markedly enriched in cancer related biological process, such as response to hypoxia, positive regulation of angiogenesis and the positive regulation of endothelial cell proliferation. KEGG pathway analysis revealed that the identified DEmRNAs were significantly enriched in the cGMP-PKG signaling pathway, cAMP signaling pathway, proteoglycans in cancer, and the regulation of actin cytoskeleton. Among these DEmRNAs, 13 mRNAs were involved in the top 10 pathways; in addition, 11 out of these 13 mRNAs exhibited positive expression correlations with lncRNA PART1 expression. RT-PCR results validated the downregulated expression levels of PART1, MYL9, KCNMA1, CHRM1, SLC25A4 and ATP1A2 in the gastric cancer compared with the adjacent normal tissues. PART1 expression has also been shown to be markedly decreased in the glioma tissues compared with normal brain tissues and its low expression was negatively associated with overall patient survival $(42,43)$. PART1 is located in chromosome $5 \mathrm{q}$, which is deleted in prostate cancer patients and cell lines and potentially contains cancer suppressor genes for prostate cancer, suggesting its crucial role in inhibiting prostate carcinogenesis $(44,45)$. The downregulation of PART1 has been shown to inhibit the cell proliferation of prostate cancer and to promote cell apoptosis by modulating the Toll-like receptor pathway (46). Bioinformatics analysis of oral squamous cell carcinoma expression profiles from the GEO database has revealed that PART1 is associated with the pathogenesis of oral squamous cell carcinoma (47). The downregulation of PART1 and its role in gastric carcinogenesis have not yet been fully elucidated. However, to the best of our knowledge, this study was the first to illustrate the PART1-enriched lncRNA-mRNA interaction network and the enriched pathway in gastric cancer. These analyses are of great significance to understanding the role of PART1 in the occurrence and development of gastric cancer. MYL9 expression has been shown to be significantly downregulated in prostate cancer tissues and its decreased expression has been shown to be negatively associated with patient survival (48). A previous study demonstrated that low MYL9 expression levels were associated with the development and metastasis of non-small cell lung cancer (49). KCNMA1 expression has been shown to be downregulated in gastric cancer cells compared with normal gastric epithelial cells and has been found to function as a tumor suppressor gene (50). SLC25A4 expression has also been shown to be downregulated during external beam radiation therapy relative to baseline and has been shown to be significantly associated with cancer-related fatigue in prostate cancer patients. SLC25A4 expression is low in proliferating cells (51). ATP1A2 expression has also been shown to be downregulated in breast cancer (52). Another study also demonstrated that ATP1A2 expression was downregulated in bone marrow-infiltrating neuroblastoma cells and was strongly associated with patient outcome (53). PART1 and many of its associated DEmiRNAs and DEmRNAs are involved in the progression of gastric cancer or other types of cancer, thereby suggesting that PART1 plays important roles in gastric cancer development.

In conclusion, the findings of this study revealed that PART1 is a DElncRNA between gastric cancer tissues and normal tissues. In this study, we generated and analyzed a PART1-mediated ceRNA network. The results indicated that PART1 interacts with DEmiRNAs (hsa-miR-4728-5p, hsa-miR-1304-3p, hsa-miR-708-5p, hsa-miR-1229-3p and hsa-miR-130b-3p) and 13 DEmRNAs. Of these, the expression levels of 11 DEmRNAs were found to be positively correlated with the PART1 expression levels. The expression levels of PART1, MYL9, KCNMA1, CHRM1, SLC25A4 and ATP1A2 were validated by RT-qPCR. The current findings of several genes, including lncRNAs, mRNAs and miRNAs, provide insight into the mechanisms underlying the pathogenesis of gastric cancer.

However, this study also had several limitations. First of all, the downregulation of PART1 needs to be verified in gastric cancer cell lines and the function of PART1 needs to be confirmed by cell function assay. Secondly, the association between candidate DEmRNAs genes and PART1 in the gastric carcinogenesis needs to be confirmed by RNA-RNA interaction assay. Despite these shortcomings, the findings of this study may prove to be of great significance for the better understanding of the role and mechanisms of action of lncRNAs in gastric cancer.

\section{Acknowledgements}

Not applicable. 


\section{Funding}

This study was supported by the Shanghai Huangpu district Municipal Health Bureau (grant no. HKM201705).

\section{Availability of data and materials}

The datasets used and/or analyzed during the current study are available from the corresponding author on reasonable request.

\section{Authors' contributions}

MJH designed the experiments, WG, JHR, XZ and XYH performed the experiments, WG and JHR analyzed the data, WG prepared the manuscript and $\mathrm{MJH}$ revised the manuscript. All authors have read and approved the final manuscript and agree to be accountable for all aspects of the research in ensuring that the accuracy or integrity of any part of the work are appropriately investigated and resolved.

\section{Ethics approval and consent to participate}

This study was approved by the Medical Ethics Committee of Ruijin Hospital Luwan Branch Shanghai Jiaotong University School of Medicine. All patients provided written informed consent.

\section{Patient consent for publication}

Not applicable.

\section{Competing interests}

The authors declare that they have no competing interests.

\section{References}

1. Overman MJ: Immunotherapy in colorectal cancer with mismatch repair deficiency. Clin Adv Hematol Oncol 17: 265-267, 2019.

2. Shariq OA, Hanson KT, McKenna NP, Kelley SR, Dozois EJ, Lightner AL, Mathis KL and Habermann EB: Does metabolic syndrome increase the risk of postoperative complications in patients undergoing colorectal cancer surgery? Dis Colon Rectum 62: 849-858, 2019.

3. Lund M, Trads M, Njor SH, Erichsen R and Andersen B: Quality indicators for screening colonoscopy and colonoscopist performance and the subsequent risk of interval colorectal cancer: A systematic review. JBI Database System Rev Implement Rep: Jun 18, 2019 (Epub ahead of print).

4. Kye BH, Rookkachart T, Kim JH, Kim HJ, Lee YS, Lee IK, Kang WK, Cho HM and Oh ST: Safety of laparoscopic oncologic resection in elderly patients with colorectal cancer: A multicenter retrospective study based on perioperative short-term outcomes. J Laparoendosc Adv Surg Tech A: Jun 12, 2019 (Epub ahead of print).

5. Kugimiya N, Harada E, Suehiro Y, Suga A, Takemoto Y and Hamano K: Determination of thymidine phosphorylase expression level facilitates recurrence risk stratification in stage II/III colorectal cancer following adjuvant chemotherapy with oral fluoropyrimidines. Oncol Lett 17: 5267-5274 2019.

6. Long B, Xiao ZN, Shang LH, Pan BY and Chai J: Impact of perioperative transfusion in patients undergoing resection of colorectal cancer liver metastases: A population-based study. World J Clin Cases 7: 1093-1102, 2019.
7. Kim MJ, Oh HK, Lee KC, Yang HH, Koo BW, Lee J, Kim MH, Kang SI, Kim DW and Kang SB: Effects of an Internet-based informational video on preoperative anxiety in patients with colorectal cancer. Ann Surg Treat Res 96: 290-295, 2019.

8. Goto N, Fukuda A, Yamaga Y, Yoshikawa T, Maruno T, Maekawa H, Inamoto S, Kawada K, Sakai Y, Miyoshi H, et al: Lineage tracing and targeting of IL17RB ${ }^{+}$tuft cell-like human colorectal cancer stem cells. Proc Natl Acad Sci USA: Jun 10, 2019 (Epub ahead of print).

9. Li K, Li L, Wu X, Yu J, Ma H, Zhang R, Li Y and Wang W: Loss of SDC1 expression is associated with poor prognosis of colorectal cancer patients in Northern China. Dis Markers 2019: 3768708 , 2019.

10. Samarakoon YM, Gunawardena NS, Pathirana A, Perera MN and Hewage SA: Prediction of colorectal cancer risk among adults in a lower middle-income country. J Gastrointest Oncol 10: 445-452, 2019.

11. Laghousi D, Jafari E, Nikbakht H, Nasiri B, Shamshirgaran M and Aminisani N: Gender differences in health-related quality of life among patients with colorectal cancer. J Gastrointest Oncol 10: 453-461, 2019.

12. Midthun L, Shaheen S, Deisch J, Senthil M, Tsai J and Hsueh CT: Concomitant KRAS and BRAF mutations in colorectal cancer. J Gastrointest Oncol 10: 577-581, 2019.

13. Taira K, Nadatani Y, Hirano S, Maeda K and Fujiwara Y: Large skin ulcer and delayed wound healing around a colostomy in a patient with metastatic colorectal cancer receiving vascular endothelial growth factor receptor-2 inhibitor therapy. Case Rep Oncol 12: 370-375, 2019

14. Amin M, Desai M, Trinkaus K, Brown A, Wang-Gillam A, Tan B, Picus J, Sorscher S, Highkin M, Lears K and Lockhart AC: Phase II trial of levocetirizine with capecitabine and bevacizumab to overcome the resistance of antiangiogenic therapies in refractory metastatic colorectal cancer. J Gastrointest Oncol 10: 412-420, 2019.

15. Aladhraei M, Al-Salami E, Poungvarin N and Suwannalert P: The roles of p53 and XPO1 on colorectal cancer progression in Yemeni patients. J Gastrointest Oncol 10: 437-444, 2019.

16. Patel A, Tripathi G, McTernan P, Gopalakrishnan K, Ali O, Spector E, Williams N and Arasaradnam RP: Fibroblast growth factor 7 signalling is disrupted in colorectal cancer and is a potential marker of field cancerisation. J Gastrointest Oncol 10: 429-436, 2019.

17. Vu JV, Matusko N, Hendren S, Regenbogen SE and Hardiman KM: Patient-reported unmet needs in colorectal cancer survivors after treatment for curative intent. Dis Colon Rectum 62: 815-822, 2019.

18. Yang C, Wei C, Wang S, Shi D, Zhang C, Lin X, Dou R and Xiong B: Elevated $\mathrm{CD} 163^{+} / \mathrm{CD} 8^{+}$ratio at tumor invasive front is closely associated with aggressive phenotype and poor prognosis in colorectal cancer. Int J Biol Sci 15: 984-998, 2019.

19. Livak KJ and Schmittgen TD: Analysis of relative gene expression data using real-time quantitative PCR and the 2(-Delta Delta C(T)) method. Methods 25: 402-408, 2001.

20. Ling A, Lundberg IV, Eklöf V, Wikberg ML, Öberg Å, Edin S and Palmqvist R: The infiltration, and prognostic importance, of Th1 lymphocytes vary in molecular subgroups of colorectal cancer. J Pathol Clin Res 2: 21-31, 2015.

21. Edelman EA and Reed EK: An update on risk assessment for familial and hereditary colorectal cancer. J Am Assoc Nurse Pract 31: 327-329, 2019.

22. Li Q, Wei L, Lin S, Chen Y, Lin J and Peng J: Synergistic effect of kaempferol and 5-fluorouracil on the growth of colorectal cancer cells by regulating the PI3K/Akt signaling pathway. Mol Med Rep, May 27, 2019 (Epub ahead of print).

23. Dahal Lamichane B, Jung SY, Yun J, Kang S, Kim DY, Lamichane S, Kim YJ, Park JH, Jang WB, Ji ST, et al: AGR2 is a target of canonical Wnt/ $\beta$-catenin signaling and is important for stemness maintenance in colorectal cancer stem cells. Biochem Biophys Res Commun, Jun 6, 2019 (Epub ahead of print).

24. Schmied BJ, Riegg F, Zekri L, Grosse-Hovest L, Bühring HJ, Jung G and Salih HR: An Fc-Optimized CD133 antibody for induction of natural killer cell reactivity against colorectal cancer. Cancers (Basel) 11: E789, 2019.

25. Cheng D, Deng J, Zhang B, He X, Meng Z, Li G, Ye H, Zheng S, Wei L, Deng X, et al: LncRNA HOTAIR epigenetically suppresses miR-122 expression in hepatocellular carcinoma via DNA methylation. EBioMedicine 36: 159-170, 2018.

26. Haddow JB, Walshe M, Aggarwal D, Thapar A, Hardman J, Wilson J, Oshowo A, Bhan C and Mukhtar H: Improving the diagnostic stage of the suspected colorectal cancer pathway: A quality improvement project. Healthc (Amst) 4: 225-234, 2016. 
27. Aziz H, Pandit V, DiGiovanni RM, Ohlson E, Gruessner AC, Jandova $J$ and Nfonsam VN: Increased incidence of early onset colorectal cancer in Arizona: A comprehensive 15-year analysis of the Arizona cancer registry. J Gastrointest Dig Syst 5: 345, 2015.

28. Guo LW, Li N, Chen HD, Lyu ZY, Feng XS, Wei LP, Li X, Wen Y, Lu M and Dai M: Progress in construction and verification of colorectal cancer risk prediction models: A systematic review. Zhonghua Yu Fang Yi Xue Za Zhi 53: 603-610, 2019 (In Chinese).

29. Nfonsam VN, Vijayasekaran A, Pandit V, E V, Aziz H, Nzuonkwelle S, Ohlson E, DiGiovanni RM and Jandova J: Patients diagnosed with colorectal cancer in rural areas in Arizona typically present with higher stage disease. J Gastrointest Dig Syst 5: pii: 346, 2015.

30. Ciombor KK and Goldberg RM: Update on anti-angiogenesis therapy in colorectal cancer. Curr Colorectal Cancer Rep 11: 378-387, 2015

31. Dong-Xu W, Jia L and Su-Juan Z: MicroRNA-185 is a novel tumor suppressor by negatively modulating the Wnt $/ \beta$-catenin pathway in human colorectal cancer. Indian $\mathrm{J}$ Cancer 52 (Suppl 3): E182-E185, 2015.

32. Du W and Fang JY: nutrients impact the pathogenesis and development of colorectal cancer. Gastrointest Tumors 2 : 203-207, 2016.

33. Wilder FG, Burnett A, Oliver J, Demyen MF and Chokshi RJ: A review of the long-term oncologic outcomes of robotic surgery versus laparoscopic surgery for colorectal cancer. Indian J Surg 78: 214-219, 2016

34. Raina K, Kumar S, Dhar D and Agarwal R: Silibinin and colorectal cancer chemoprevention: A comprehensive review on mechanisms and efficacy. J Biomed Res 30: 452-465, 2016.

35. Jia H, Xiang L, Wang Z and Zhou Q: A study on the mechanism of low-expressed cancer stem cell marker Lgr5 in inhibition of the proliferation and invasion of colorectal carcinoma. Cell Biochem Biophys 73: 393-397, 2015.

36. Urbanska AM,Zhang X and Prakash S: Bioengineered colorectal cancer drugs: Orally delivered anti-inflammatory agents. Cell Biochem Biophys 72: 757-769, 2015.

37. Ahronian LG and Corcoran RB: Effective MAPK Inhibition is critical for therapeutic responses in colorectal cancer with BRAF mutations. Mol Cell Oncol 3: e1048405, 2016.

38. Sheng QS, Hua HJ, Cheng XB, Wang WB, Chen WB, Xu JH and Lin JJ: A modified spontaneously closed defunctioning tube ileostomy after anterior resection of the rectum for rectal cancer with a low colorectal anastomosis. Indian J Surg 78: 125-129, 2016.

39. Han YH, Lim ST, Jeong HJ and Sohn MH: Clinical value of a one-stop-shop low-dose lung screening combined with (18) F-FDG PET/CT for the detection of metastatic lung nodules from colorectal cancer. Nucl Med Mol Imaging 50: 144-149, 2016.

40. Triantafillidis JK, Vagianos C and Malgarinos G: Colonoscopy in colorectal cancer screening: Current aspects. Indian J Surg Oncol 6: 237-250, 2015

41. Dawood S, Sirohi B, Shrikhande SV, Toh HC and Eng C: Potential prognostic impact of baseline CEA level and surgery of primary tumor among patients with synchronous stage IV colorectal cancer: A large population based study. Indian J Surg Oncol 6: 198-206, 2015.
42. Koelzer VH, Canonica K, Dawson H, Sokol L, KaramitopoulouDiamantis E, Lugli A and Zlobec I: Phenotyping of tumor-associated macrophages in colorectal cancer: Impact on single cell invasion (tumor budding) and clinicopathological outcome. Oncoimmunology 5: e1106677, 2015.

43. Correale P, Botta C, Martino EC, Ulivieri C, Battaglia G, Carfagno T, Rossetti MG, Fioravanti A, Guidelli GM, Cheleschi S, et al: Phase Ib study of poly-epitope peptide vaccination to thymidylate synthase (TSPP) and GOLFIG chemo-immunotherapy for treatment of metastatic colorectal cancer patients. Oncoimmunology 5: e1101205, 2015.

44. Rosa I, Fidalgo P, Filipe B, Albuquerque C, Fonseca R, Chaves P and Pereira AD: Sporadic colorectal cancer: Studying ways to an end. United European Gastroenterol J 4: 288-296, 2016.

45. Meyerhardt JA: What should we tell our patients with colorectal cancer about diet, supplements, and lifestyle? Clin Adv Hematol Oncol 13: 636-638, 2015.

46. Sun M, Geng D, Li S, Chen Z and Zhao W: LncRNA PART modulates toll-like receptor pathways to influence cell proliferation and apoptosis in prostate cancer cells. Biol Chem 399 387-395, 2018.

47. Angelova M, Charoentong P, Hackl H and Trajanoski Z: The colorectal cancer immune paradox revisited. Oncoimmunology 5: e1078058, 2015.

48. Jurjus A, Eid A, Al Kattar S, Zeenny MN, Gerges-Geagea A, Haydar H, Hilal A, Oueidat D, Matar M, Tawilah J, et al: Inflammatory bowel disease, colorectal cancer and type 2 diabetes mellitus: The links. BBA Clin 5: 16-24, 2015.

49. Sokolova EA, Boyarskikh UA, Shirshova AN, Kel AE and Filipenko ML: The biomarkers for timely diagnostics of colorectal cancer. Klin Lab Diagn 60: 15-23, 2015 (In Russian).

50. Echterdiek F, Janikovits J, Staffa L, Müller M, Lahrmann B, Frühschütz M,Hartog B, Nelius N, Benner A, Tariverdian M, et al: Low density of FOXP3-positive T cells in normal colonic mucosa is related to the presence of beta2-microglobulin mutations in Lynch syndrome-associated colorectal cancer. Oncoimmunology 5: e1075692, 2015.

51. Russo G, Patrignani A, Poveda L, Hoehn F, Scholtka B, Schlapbach R and Garvin AM: Highly sensitive, non-invasive detection of colorectal cancer mutations using single molecule, third generation sequencing. Appl Transl Genom 7: 32-39, 2015.

52. Stoll G,Zitvogel L and KroemerG: Differences in the composition of the immune infiltrate in breast cancer, colorectal carcinoma, melanoma and non-small cell lung cancer: A microarray-based meta-analysis. Oncoimmunology 5: e1067746, 2015.

53. Lu LL, Chen XH, Zhang G, Liu ZC, Wu N, Wang H, Qi YF, Wang HS, Cai SH and Du J: CCL21 facilitates chemoresistance and cancer stem cell-like properties of colorectal cancer cells through AKT/GSK-3 $\beta /$ Snail signals. Oxid Med Cell Longev 2016: 5874127, 2016.

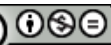

This work is licensed under a Creative Commons Attribution-NonCommercial-NoDerivatives 4.0 International (CC BY-NC-ND 4.0) License. 